\title{
Cytotoxic drug sensitivity of Epstein-Barr virus transformed lymphoblastoid B-cells
}

\author{
Laszlo Markasz 1,2, György Stuber ${ }^{1}$, Emilie Flaberg1 1 Åsa Gustafsson Jernberg3 , \\ Staffan Eksborg ${ }^{4}$, Eva Olah², Henriette Skribek ${ }^{1}$ and Laszlo Szekely*1
}

\begin{abstract}
Address: ${ }^{1}$ Department of Microbiology, Tumor and Cell Biology (MTC) and Center for Integrative Recognition in the Immune System (IRIS), Karolinska Institute, Box 280 S-17177 Stockholm, Sweden, ${ }^{2}$ Department of Pediatrics University of Debrecen, Medical and Health Science Center, Debrecen, Hungary, ${ }^{3}$ Department of Pediatrics, Karolinska Institutet, Karolinska University Hospital, Huddinge, Sweden and ${ }^{4}$ Karolinska Pharmacy, and Department of Woman and Child Health, Childhood Cancer Research Unit, Karolinska Institutet, Karolinska University Hospital, Stockholm, Sweden

Email: Laszlo Markasz - markaszl@freemail.hu; György Stuber - Gyorgy.Stuber@ki.se; Emilie Flaberg - Emilie.Flaberg@ki.se;

Åsa Gustafsson Jernberg - Asa.Gustafsson.Jernberg@ki.se; Staffan Eksborg - staffan.eksborg@karolinska.se; Eva Olah - olah_e@gyermek.dote.hu; Henriette Skribek - Henriette.Skribek@ki.se; Laszlo Szekely* - lassze@ki.se

* Corresponding author
\end{abstract}

Published: 13 November 2006

BMC Cancer 2006, 6:265 doi:10.1 I86/147/-2407-6-265
Received: 30 August 2006

Accepted: 13 November 2006

This article is available from: http://www.biomedcentral.com/I47I-2407/6/265

(C) 2006 Markasz et al; licensee BioMed Central Ltd.

This is an Open Access article distributed under the terms of the Creative Commons Attribution License (http://creativecommons.org/licenses/by/2.0), which permits unrestricted use, distribution, and reproduction in any medium, provided the original work is properly cited.

\begin{abstract}
Background: Epstein-Barr virus (EBV) is the causative agent of immunosuppression associated lymphoproliferations such as post-transplant lymphoproliferative disorder (PTLD), AIDS related immunoblastic lymphomas (ARL) and immunoblastic lymphomas in X-linked lymphoproliferative syndrome (XLP). The reported overall mortality for PTLD often exceeds $50 \%$. Reducing the immunosuppression in recipients of solid organ transplants (SOT) or using highly active antiretroviral therapy in AIDS patients leads to complete remission in $23-50 \%$ of the PTLD/ARL cases but will not suffice for recipients of bone marrow grafts. An additional therapeutic alternative is the treatment with anti-CD20 antibodies (Rituximab) or EBV-specific cytotoxic T-cells. Chemotherapy is used for the non-responding cases only as the second or third line of treatment. The most frequently used chemotherapy regimens originate from the non-Hodgkin lymphoma protocols and there are no cytotoxic drugs that have been specifically selected against EBV induced lymphoproliferative disorders.

Methods: As lymphoblastoid cell lines (LCLs) are well established in vitro models for PTLD, we have assessed 17 LCLs for cytotoxic drug sensitivity. After three days of incubation, live and dead cells were differentially stained using fluorescent dyes. The precise numbers of live and dead cells were determined using a custom designed automated laser confocal fluorescent microscope.
\end{abstract}

Results: Independently of their origin, LCLs showed very similar drug sensitivity patterns against 29 frequently used cytostatic drugs. LCLs were highly sensitive for vincristine, methotrexate, epirubicin and paclitaxel.

Conclusion: Our data shows that the inclusion of epirubicin and paclitaxel into chemotherapy protocols against PTLD may be justified. 


\section{Background}

Development of malignant B-cell lymphomas after organ transplantation is a significant complication arising as a side effect of the immunosuppression required for successful graft survival. The oncogenic Epstein-Barr virus (EBV) is the etiologic agent in the posttransplant lymphoproliferative disorder (PTLD) and AIDS related immunoblastic lymphomas (ARL) [1]. The reported overall mortality for PTLD often exceeds 50\% [2,3]. The prognosis for PTLDs occurring after bone marrow transplantation is even worse $[4,5]$. Male patients with the rare inherited $\mathrm{X}$-linked lymphoproliferative syndrome, showing specific immune defect against EBV infection, also often succumb to EBV induced malignant lymphomas [6].

EBV is a ubiquitous human herpesvirus that persists for life. Primary EBV infection can lead to mononucleosis (IM) in adolescence and in adults, manifested by a massive expansion of B cells. EBV-encoded transformationassociated proteins drive the proliferation of $\mathrm{B}$ lymphoblasts in IM, in PTLDs and in immunodeficiency syndrome-associated immunoblastic lymphomas. The EBV transformed cells express nine latency-associated viral proteins: EBNA1-6, LMP-1, -2A and -2B. This latency program is referred to as the type III latency. The same latency program is present in the in vitro proliferating lymphoblastoid cell lines (LCLs), generated by infection of normal human B cells with EBV. The fraction of B cells that is susceptible to in vitro transformation can be anything between $10 \%$ and $100 \%$ [7].

EBV drives the proliferation of human B cells in vitro and during primary infection in vivo. Strong T cell-mediated immune responses have been documented against EBV encoded latent proteins and a wide range of HLA class I molecules with EBV originated peptide epitopes have been identified [8-10]. EBV associated lymphoproliferative disease can develop only in the absence of a competent cytotoxic T cell immune surveillance. EBV associated lymphoproliferative disease may disappear upon treatment restoring the immune response against EBV-infected B cells.

Historically LCLs were often regarded as non-tumorigenic in immunosupressed mice upon subcutaneous inoculation, especially in comparison with highly tumorigenic Burkitt's lymphomas. However intraperitoneal inoculation regularly leads to development of generalized lymphomas with multiorgan involvement. SCID mice inoculated intraperitoneally with peripheral blood lymphocytes (PBL) from EBV-seropositive donors or with human LCLs, develop EBV-induced human lymphoproliferations within a few weeks. These lymphomas are classified as immunoblastic lymphomas, often with plasmacytoid features [11]. Histologically the PBL derived
human-SCID tumors very much resemble the EBV positive large cell lymphomas of immunosuppressed patients [12]. The tumors of the immunocompromised patients or the experimental tumors growing in immunodefective mice as well as the in vitro growing LCLs show very similar phenotypes. All three express the same spectrum of cell surface markers, B cell activation antigens and adhesion molecules. All three have normal karyotype and show identical viral gene expression patterns.

The risk of PTLD has been found to depend upon the type of the transplanted organ, the immunosuppressive regimen, the age, the underlying illness and the EBV status of the recipient at the time of transplantation. The estimated incidence of PTLD ranges from 1-4\% after renal transplantation to $19 \%$ after intestinal transplantation. In bone marrow allograft recipients PTLD is relatively uncommon $(1 \%)[13,14]$ except for when certain high risk regimes, such as in vitro T-cell depletion (TCD) are used, when the risk may rise to $30 \%[4,15]$ PTLD following allogenic stem cell transplantation usually derives from donor lymphocytes. The risk of PTLD is greater if the host is EBV-seronegative at the time of transplantation and/or if there is a mismatch between the donor and recipient HLA types [1].

No controlled studies have been performed in the management of PTLD and most of the recommendations for therapy come from small cohorts at single institutions [1]. The relative importance of $\mathrm{T}$ cell impairment, EBV and clonal proliferation has led to the following strategies: reduction of immunosuppression or prophylactic restoration of T-cell immunity [16], antiviral therapy and chemotherapy. Reducing the immunosuppression leads to complete and durable remission of PTLD [17] for 23$50 \%$ of patients after organ transplantation (SOD) but will not be efficient in the BMT setting. Reduction of immunosuppression is frequently the first therapeutic step, and patients who have had organ rejection have a much poorer prognosis.

For secondary EBV lymphomas post BMT none of the above regimens will suffice, except when specifically restoring EBV specific immunity [16]. Anti-B-cell monoclonal antibodies are an effective therapy for PTLD. A combination of anti CD21 and anti-CD24 antibodies were used. However these antibodies are not commercially available and interest has therefore recently turned towards Rituximab, a humanized monoclonal antibody against the pan-B cell marker CD20. The response rate of Rituximab treatment is $65 \%$ with a relapse rate of $18 \%$ $[18,19]$. 
In cases that are not responding to the above mentioned therapy, chemotherapy is used as the second or third line of option.

The most frequently used chemotherapy protocols are the anthracycline based CHOP (cyclophosphamide, doxorubicin, vincristine and prednisolone) and VAPEC-B (doxorubicin, etoposide, cyclophosphamide, methotrexate, bleomycin, and vincristine) [20]. In the study of Muti et al. 17 of 40 PTLD patients were treated with a combination of reduced immunosuppression and chemotherapy (CHOP, VACOP-B - cyclophosphamide, vincristine, bleomycin, prednisolone, with increasing doses of doxorubicin and etoposide- or DHAP -cisplatinum, cytosinearabinoside, dexamethasone) [21]. Remission rate for anthracycline-based combination therapy is $69 \%$.

PTLD patients are exposed to a high mortality risk due to chemotherapy treatment related toxicity [1].

The side effects of a drug are the limiting factor in determining the dose. Increasing the dose of a drug leads to a more frequent appearance of side effects. Chemotherapeutic agents can also compromise the survival of the graft. Drugs that are effective even at low doses could therefore, not only give fewer side effects, but also provide a better graft survival.

The aim of this study was to find the most effective cytotoxic drugs against EBV-induced lymphoproliferations. We carried out over 6500 cell survival and proliferation assays, on single cell level, in order to establish the pattern of in vitro efficacy of the most commonly used cytotoxic drugs against EBV-transformed lymphoblastoid cells.

\section{Methods}

\section{Cell lines and culture conditions}

17 lymphoproliferative cell lines (LCLs) were used in the present study. 980215, 031016, 040113, 051018, JAK, LP, MIN, AF, GK, FUR, HA, VMB were established by in vitro infection of normal B lymphocytes, from different healthy donors, with the B95.8 strain of EBV. These cell lines were expanded in IMDM (Sigma) medium supplemented with $10 \%$ fetal calf serum (FCS). LSspontan is a spontaneous LCL established from a healthy donor. IARC171 was established using B95.8 virus infection of normal lymphocytes from a patient with Burkitt's lymphoma. TR was established from B cells of an XLP patient, with deletion in the SAP gene. The cell line IB4 contains an integrated EBV genome.

During the time of experiment the LCLs were cultured in IMDM (Sigma) supplemented with 20\% FCS (Sigma), $100 \mathrm{mmol}$ L-glutamine (Sigma) and $80 \mu \mathrm{g} / \mathrm{ml}$ gentamicin (Sigma). Cell suspensions were grown in a humidified incubator at $37^{\circ} \mathrm{C}$ in an atmosphere containing $5 \% \mathrm{CO}_{2}$. Cell counts were adjusted to an optimal concentration of $1 \times 10^{6} \mathrm{cells} / \mathrm{ml}$ and the cells were fed twice a week. The absence of mycoplasma contamination was assured by regular monitoring with Hoechst 33258 staining.

\section{Drugs}

For the in vitro drug sensitivity test 29 drugs were used (summarized in Table 1). All the drugs were dissolved in $50 \%$ dimethyl sulfoxide (DMSO) and printed on 384 well plates, using a high density metal pin array (with $50 \mathrm{nl}$ replica volumes) in Biomek 2000 fluid dispenser robot (Beckman). The same robot was used to generate the drug masterplates containing the triplicates of four different drug dilutions using a single tip automatic dispenser head.

The highest drug concentration was selected as the physicochemical maximally achievable drug concentration at 600 times dilution (50 $\mathrm{nl}$ drug in $30 \mu \mathrm{l}$ suspension assay volume)

The Ratio of Maximum Achieved Plasma Concentration (RMAPC) was determined for each drug concentration in order to compare the in vitro tested concentrations to the in vivo maximally achieved plasma concentration (Cmax) levels.

RMAPC was calculated according to the following equation:

$\mathrm{RMAPC}=$ in vitro tested concentration/Cmax

\section{In vitro drug sensitivity assay}

In vitro drug sensitivity of LCLs was assessed using a 3-day cell culture on microtiter plates. 28 drugs were tested, each at 4 different concentrations in triplicates on 384-well plates (Greiner). Each well was loaded with $30 \mu \mathrm{l}$ cell suspension containing 3000 cells. After three days of incubation the live and dead cells were differentially stained using fluorescent VitalDye (Biomarker, Hungary). The exact number of live and dead cells was determined using a custom designed automated laser confocal fluorescent microscope (a modified Perkin-Elmer UltraView LCI system) at the Karolinska Institute visualization core facility (KIVIF). The images were captured using the computer program QuantCapture 4.0 and the live and dead cells were identified and individually counted using the program QuantCount 3.0. Both programs were developed at the KIVIF using OpenLab Automator programming environment (Improvision). 15 control wells, that were used to determine the control cell survival (CCS), contained cells with only culture medium and $50 \mathrm{nl}$ DMSO without drugs, 5 wells contained cells with culture medium alone. Comparing the two types of control wells no toxic effect 
$\stackrel{\text { ¿ }}{\circlearrowright}$ Table I: Used drugs, Cmax, AUC levels

\begin{tabular}{|c|c|c|c|c|c|c|c|c|}
\hline Drug groups & Drug name & Brand name, company & $\begin{array}{c}\text { In vitro used } \\
\text { concentrations } \\
(\mu \mathrm{g} / \mathrm{ml})\end{array}$ & $\underset{(\mu \mathrm{g} / \mathrm{ml})}{\mathrm{Cmax} \text { Value }}$ & $\begin{array}{l}\text { In vivo dose } \\
(\text { Cmax })\end{array}$ & $\begin{array}{c}\text { AUC } \\
(\mu \mathrm{g} \times \mathrm{h} / \mathrm{ml})\end{array}$ & $\begin{array}{l}\text { In vivo dose } \\
\text { (AUC) }\end{array}$ & Reference \\
\hline \multirow[t]{3}{*}{ anthracyclines } & epirubicin & Epirubicin Meda, Meda & $0.026-1.66$ & 9 & $120 \mathrm{mg} / \mathrm{m}^{2}$ & 2.412 & $90 \mathrm{mg} / \mathrm{m}^{2}$ & (Fogli et al. 2002) [23] \\
\hline & daunorubicin & Cerubidin ${ }^{\circledR}$, Aventis Pharma & $0.065-4.166$ & 0.49 & $0.95 \mathrm{mg} / \mathrm{kg}$ in $45 \mathrm{~min}$ & 1.2786 & $1.5 \mathrm{mg} / \mathrm{kg}$ & (Andersson et al. 1979) [6] \\
\hline & doxorubicin & Doxorubicin Teva, Teva & $0.026-1.66$ & 0.93 & $50 \mathrm{mg} / \mathrm{m}^{2}$ & 0.82464 & $50 \mathrm{mg} / \mathrm{m}^{2}$ & (Toffoli et al. 2004) [24] \\
\hline epipodophyllotoxin & etoposide & Sigma & $0.325-20.833$ & 2.5 & $53 \mathrm{mg} / \mathrm{m}^{2} /$ day & 5.06 & $100 \mathrm{mg} / \mathrm{m}^{2} /$ day & (Minami et al. 1995) ${ }_{[26]}$ (Gruber et al. 1995) \\
\hline camptothecins & topotecan & Hycamtin $^{\circledR}$, GlaxoSmithKline & $0.052-3.33$ & 0.0084 & $2.3 \mathrm{mg} / \mathrm{m}^{2} /$ day & 0.0196 & $1.2 \mathrm{mg} / \mathrm{m}^{2} /$ day & (Gerrits et al. 1999) [27] \\
\hline \multirow{2}{*}{ taxanes } & paclitaxel & Taxol, Orifarm & $0.078-5$ & 3.38 & $175 \mathrm{mg} / \mathrm{m}^{2}$ by $3 \mathrm{~h}$ & 13.49 & $175 \mathrm{mg} / \mathrm{m}^{2}$ & (Fogli et al. 2002) [23] \\
\hline & docetaxel & Taxotere ${ }^{\circledR}$, Aventis Pharma & $0.52-33.33$ & 2 & $60 \mathrm{mg} / \mathrm{m}^{2}$ & 3.326 & $85 \mathrm{mg} / \mathrm{m}^{2}$ & (Rischin et al. 2002) [28] \\
\hline \multirow[t]{3}{*}{ vinca alkaloids } & vincristine & Vincristine Mayne, Mayne Pharma & $0.0065-0.416$ & 0.37 & $1.4 \mathrm{mg} / \mathrm{m}^{2}$ & 0.182 & $1.32 \mathrm{mg} / \mathrm{m}^{2}$ & $\begin{array}{c}\text { (Terashi et al. 2000) [29] (Desai et al. 1982) } \\
{[30]}\end{array}$ \\
\hline & vinblastine & Velbe ${ }^{\circledR}$, STADApharm & $0.013-0.833$ & 0.005 & $1.5 \mathrm{mg} / \mathrm{m}^{2}$ by $24 \mathrm{~h}$ & 0.218 & $1.7 \mathrm{mg} / \mathrm{m}^{2}$ & (Stewart et al. 1983) [3I] (Bates et al.) [32] \\
\hline & vinorelbine & Navelbine ${ }^{\circledR}$, Pierre Fabre & $0.13-8.33$ & 0.83 & $200 \mathrm{mg} /$ week & 0.899 & $80 \mathrm{mg} / \mathrm{m}^{2} /$ week & $\begin{array}{c}\text { (Zhou et al. 1991) [33] (Freyer et al. 2003) } \\
\text { [34] }\end{array}$ \\
\hline \multirow[t]{2}{*}{ platinum analogs } & carboplatin & Carboplatin Mayne, Mayne Pharma & $0.13-8.33$ & 0.046 & $360 \mathrm{mg} / \mathrm{m}^{2}$ & 348000 & $360 \mathrm{mg} / \mathrm{m}^{2}$ & (Ghazal-Aswad et al. 1996) [35] \\
\hline & oxaliplatin & Eloxatin, Sanofi-Synthelabo & $0.065-4.166$ & 3.2 & $130 \mathrm{mg} / \mathrm{m}^{2}$ & 71.5 & $130 \mathrm{mg} / \mathrm{m}^{2}$ & $\begin{array}{l}\text { (Gamelin et al. 1997) [36] (Graham et al. } \\
\text { 2000) [37] }\end{array}$ \\
\hline antifolate & methotrexate & Methotrexate Pharmacia, Pfizer & $0.325-20.833$ & 363 & $8-12 \mathrm{~g} / \mathrm{m}^{2}$ & 13200000 & $12 \mathrm{~g} / \mathrm{m}^{2}$ & (Bacci et al. 2006) [38] (Crews et al. 2004) \\
\hline \multirow[t]{2}{*}{ purin antagonists } & 6-mercaptopurine & Sigma & $1.085-69.44$ & 2 & $2.5 \mathrm{mg} / \mathrm{kg}$ oral dose & 0.2587 & $85 \mathrm{mg} / \mathrm{m}^{2}$ & (Chan et al. 1989) [26] \\
\hline & cladribine & Leustatin, Janssen-Cilag & $0.013-0.833$ & 0.0356 & $5 \mathrm{mg} / \mathrm{m}^{2}$ & 0.1541 & $5 \mathrm{mg} / \mathrm{m}^{2}$ & (Albertioni et al. 1998) [40] \\
\hline \multirow[t]{3}{*}{ pyrimidine antagonists } & fluorouracil & Fluorouracil Mayne, Mayne Pharma & $0.65-41.66$ & 55.4 & $400 \mathrm{mg} / \mathrm{m}^{2}$ & 11.59 & $400 \mathrm{mg} / \mathrm{m}^{2}$ & (Casale et al. 2004) [4I] \\
\hline & cytarabine & Cytarabine Pfizer, Pfizer & $1.302-83.33$ & 10.8 & $3 \mathrm{~g} / \mathrm{m}^{2}$ & 523.4 & $\mathrm{I} / \mathrm{m}^{2}$ & $\begin{array}{c}\text { (DeAngelis et al. 1992) [42] (Gruber et al. } \\
\text { 1995) [26] }\end{array}$ \\
\hline & gemcitabine & Gemzar, Orifarm & $0.781-50$ & 22.3 & $1000 \mathrm{mg} / \mathrm{m}^{2}$ & 9.3 & $1000 \mathrm{mg} / \mathrm{m}^{2}$ & (Fogli et al. 2002) [23] \\
\hline \multirow[t]{2}{*}{ antitumor antibiotics } & bleomycin & Bleomycin Baxter, Baxter & $0.39-25^{*}$ & $0.19 *$ & $30 \mathrm{lU} / \mathrm{day}$ & 0.089 & $8 \mathrm{IU} / \mathrm{kg} /$ day & $\begin{array}{c}\text { (Broughton et al. 1977) [43] (Peng et al. 1980) } \\
\text { [44] }\end{array}$ \\
\hline & dactinomycin & Cosmegen $^{\circledR}$, MSD & $0.006-0.416$ & 0.1 & $1.5 \mathrm{mg} / \mathrm{m}^{2}$ & 300 & $1.5 \mathrm{mg} / \mathrm{m}^{2}$ & (Veal et al. 2005) [45] \\
\hline protease inhibitors & $\begin{array}{l}\text { bortezomib } \\
\text { MGI32 }\end{array}$ & $\begin{array}{l}\text { Velcade }^{\circledR}, \text { Janssen-Cilag } \\
\text { Sigma }\end{array}$ & $\begin{array}{l}0.013-0.833 \\
0.13-8.33\end{array}$ & $\begin{array}{c}0.08 \\
\#\end{array}$ & $1.45 \mathrm{mg} / \mathrm{m}^{2}$ & 0.0438 & $1.45 \mathrm{mg} / \mathrm{m}^{2}$ & (Papandreou et al. 2004) [46] \\
\hline \multirow{4}{*}{ alkylating agents } & cyclophosphamide & Sendoxan, Baxter & $0.52-33.33$ & 37.2 & $50 \mathrm{mg} / \mathrm{kg}$ & 367 & $50 \mathrm{mg} / \mathrm{kg}$ & (Xie et al. 2006) [47] \\
\hline & ifosphamide & Holoxan ${ }^{\circledR}$, Baxter & $0.52-33.33$ & 56.63 & $16 \mathrm{~g} / \mathrm{m}^{2}$ in $96 \mathrm{~h}$ & 1827.7 & $3 \mathrm{~g} / \mathrm{m}^{2} /$ day & $\begin{array}{c}\text { (Wright et al. 1995) [48] (Boddy et al. 1996) } \\
{[45]}\end{array}$ \\
\hline & chlorambucil & Sigma & $1.302-83.33$ & 0.49 & $0.2 \mathrm{mg} / \mathrm{m}^{2}$ & 0.883 & $0.2 \mathrm{mg} / \mathrm{m}^{2}$ & $\begin{array}{c}\text { (GlaxoSmithKline Research Triangle Park) } \\
\text { [49] }\end{array}$ \\
\hline & streptozotocin & Sigma & $0.65-41.66$ & $\mathfrak{a}$ & & & & \\
\hline \multirow[t]{3}{*}{ miscellaneous } & asparaginase & Asparaginase Medac, Medac & $0.065-4.166^{*}$ & $4.48^{*}$ & $10000 \mathrm{IU} / \mathrm{m}^{2}$ & 0.943 & $30000 \mathrm{IU} / \mathrm{m}^{2}$ & $\begin{array}{c}\text { (Ho et al. 198I) }[50] \text { (Ylikangas et al. 2002) } \\
{[5 \mathrm{I}]}\end{array}$ \\
\hline & hydroxyurea & Sigma & $0.65-41.66$ & 21 & $18 \mathrm{mg} / \mathrm{kg}$ & 82.49 & $15 \mathrm{mg} / \mathrm{kg}$ & (Gwilt et al. 2003) [52] (Yan et al. 2005) [46] \\
\hline & prednisolone & Di-Adreson F Aquosum, Organon & $1.56-100$ & 0.325 & $\mathrm{l} \mathrm{mg} / \mathrm{kg} / \mathrm{day}$ & 1.33 & $\mathrm{I} \mathrm{mg} / \mathrm{kg} / \mathrm{day}$ & (Rostin et al. 1990) [53] \\
\hline
\end{tabular}

Cmax: maximum plasma level after administration of the clinical dose of the drug

*In case of bleomycin and asparaginase concentrations are in IU/ml

AUC: area under curve

\#: not used in the clinical practice

x: no data is available 
of DMSO could be seen. Mean cell survival (MCS) was determined from the average of cell survival of all 17 LCLs.

\section{Results \\ Comparison of the drug sensitivity pattern of the different LCLs}

The 17 different LCLs that were tested in the present study represent a large variety of cells with different origins and in vitro history. The investigation included cell lines with several years of continuous in vitro culturing along with freshly established transformed B-cell cultures of only 3 weeks of age. Many of the lines were transformed by the B95-8 strain of EBV and some of them were spontaneous outgrowths driven by the donors own virus. Among the lines, we had LCLs from healthy donors, from XLP patients or lines established from the normal B-cells of Burkitt's lymphoma patients. We tested lines with both episomal and integrated EBV genome.

Remarkably, the LCLs of different origins showed a very similar sensitivity pattern against the different drugs. This phenomenon is illustrated in Figure 1, which summarizes the survival curves of all 17 LCLs against the four different concentrations of epirubicin. Each individual thin curve represents the average of three independent measurements. The thick line is the mean of all curves, the Mean Cell Survival (MCS). The grey shaded area marks the \pm standard deviation (SD). Figure 2 summarizes the Mean Cell Survival curve along with the standard deviations for 28 drugs again emphasizing the very similar response of the different cell lines. The values for MG132 are represented only in Table 2.

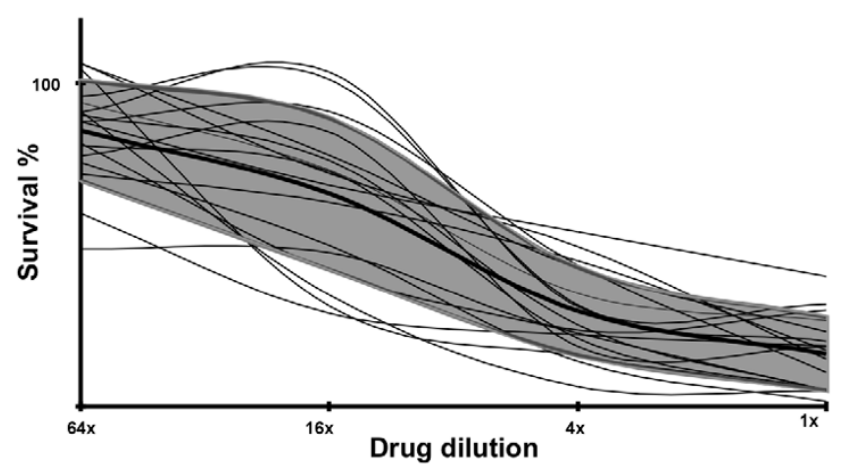

Figure I

Drug sensitivity pattern of 17 lymphoblastoid cell lines for epirubicin. The mean value of survival of LCLs is represented by the black curve, $\pm S D$ value is marked with grey. I $x$ : highest concentration, 16x: 16 times dilution of the highest concentration etc.

\section{Identification of highly effective and non-effective drugs}

The starting concentrations of the dilution series $(1 \times, 4 \times$, $16 \times, 64 \times)$ for the individual drugs were initially determined based on the solubility of the different agents. To make the cell survival data comparable with each other, we first compiled the known Maximum Achieved Plasma Concentration data (Cmax) for all 28 drugs stated from the literature (Table 1-column 5). Dividing the actual drug concentrations in the dilution series with the Cmax of the particular drug yielded the Ratio of Maximum Achieved Plasma Concentration (RMAPC). The calculated values of RMAPC for all the drugs are summarized in Table 2 along with MCS and \pm SD values. Plotting the cell survival against the RMAPC values allowed a direct comparison of the effectiveness of different drugs. Figure 3 shows the comparison of the effectiveness of the members of three different drug families: the anthracyclines, vinca alcaloids and taxans. Figure 4 summarizes the mean cell survival curves for all the drugs along a common RMAPC axis. Based on their effectiveness on LCLs, we have divided the drugs into three different groups:

Group 1: The drugs were considered to be highly effective if the mean cell survival (MCS) was below 30\% at RMAPC 0.3 or below that.

Group 2: The drug was partially effective if MCS was under 60\% and RMAPC was between 0.3 and 1 .

Group 3: The drug was ineffective, e.g. the LCLs were resistant to the drug, if MCS was above $60 \%$ or RMAPC was above 1 .

We found that the four most effective drugs against LCLs were: vincristine, paclitaxel, methotrexate and epirubicin (Group 1).

Gemcitabin, cytosine-arabinoside, doxorubicin, fluorouracil, dactinomycin, docetaxel, daunorubicin, etoposide, vinorelbine was rated as partially effective (Group 2.)

Most LCLs were resistant to cyclophosphamide, asparaginase, topotecan, oxaliplatin, bleomycin, 6-mercaptopurin, hydroxyurea, cladribine, chlorambucil, carboplatin, bortezomib, cytarabine, prednisolone and vinblastine (Group 3). Almost no drug response could be seen with oxaliplatin, bleomycin, cyclophosphamide, asparaginase, hydroxyurea and ifosphamide whereas vinblastine, chlorambucil, prednisolone and topotecan were effective, but only at very high concentrations, well above the maximum achievable plasma concentrations.

Although the Group 3 drugs were not effective against LCLs, these drugs show concentration dependent growth 

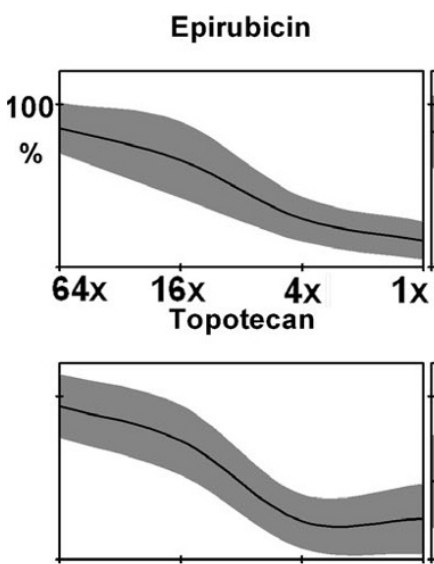

Vinblastine

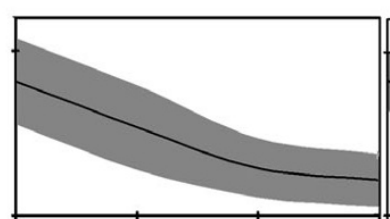

Methotrexate

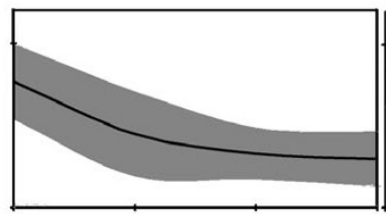

Cytarabine

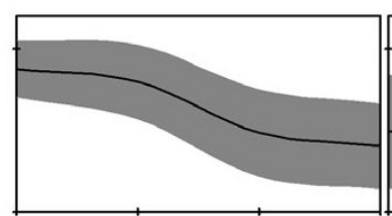

Bortezomib

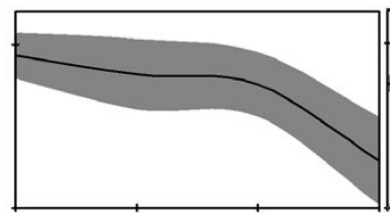

Chlorambucil

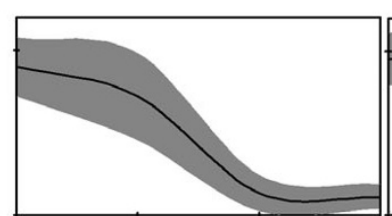

Daunorubicin

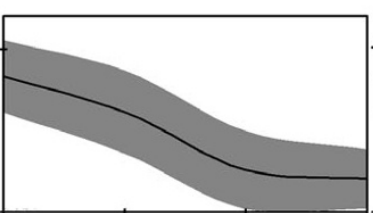

Paclitaxel

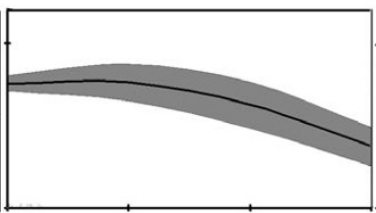

Streptozotocin

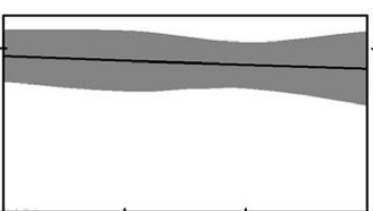

Doxorubicin

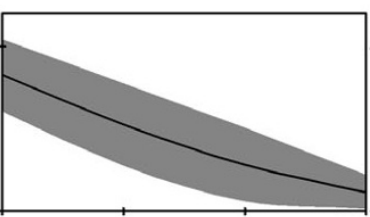

Docetaxel

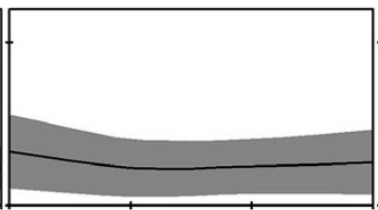

Carboplatin

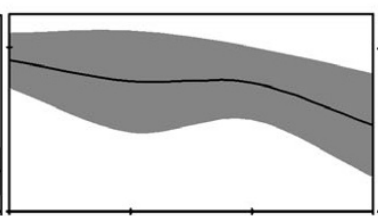

Cladribine

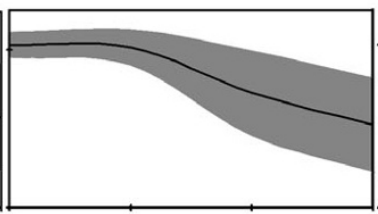

Bleomycin

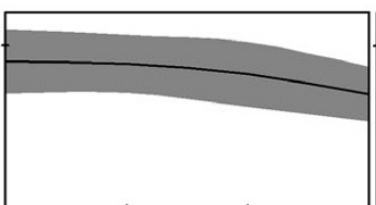

Cyclophosphamide

Etoposide

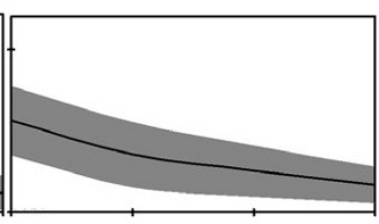

Vincristine

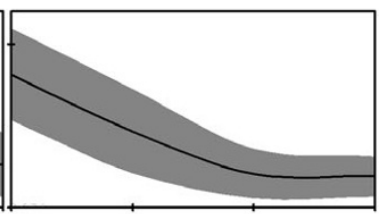

Oxaliplatin

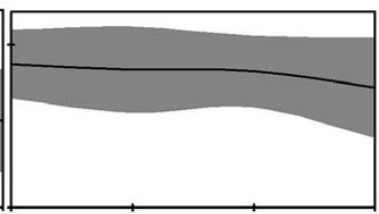

Fluorouracil

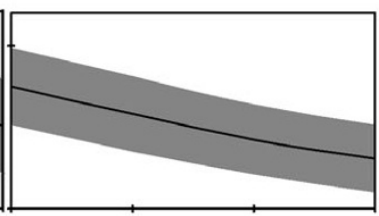

Dactinomycin

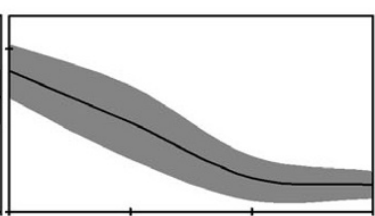

Ifosphamide

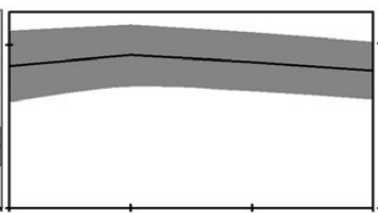

Asparaginase

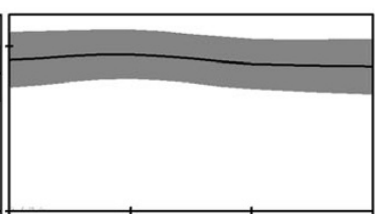

Hydroxyurea
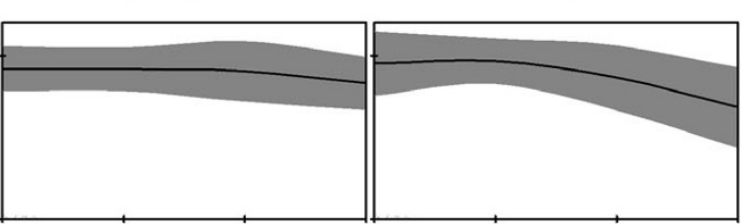

Figure 2

Mean values and standard deviations of drug sensitivity of 17 lymphoblastoid cell lines for 28 different cytostatic drugs. $y$ axis: fraction of surviving cells $0-100 \% \times$ axis: drug dilutions ( $1 \times$ represents the highest concentration). 
Table 2: The average Mean Cell Survival (MCS) of the 17 LCLs at different drug concentrations, expressed as the Ratio of Maximum Achieved Plasma Concentration (RMAPC)

\begin{tabular}{|c|c|c|c|c|c|c|c|c|c|c|}
\hline & & $64 \times$ dilution & $16 \times$ dilution & $4 \times$ dilution & I $\times$ no dilution & & $64 \times$ dilution & $16 \times$ dilution & $4 \times$ dilution & $\mathrm{I} \times$ no dilution \\
\hline \multirow[t]{8}{*}{ Group I } & \multicolumn{5}{|l|}{ Vincristine } & \multicolumn{5}{|l|}{ Epirubicin } \\
\hline & RMAPC & 0.018 & 0.071 & 0.282 & 1.128 & RMAPC & 0.003 & 0.012 & 0.046 & 0.184 \\
\hline & MCS & 81.498 & $46.54 I$ & 20.776 & 18.995 & MCS & 85.206 & 65.736 & 29.435 & 16.306 \\
\hline & SD & 27.995 & 25.737 & 15.258 & 12.209 & SD & 15.548 & 23.394 & 13.593 & 11.314 \\
\hline & \multicolumn{5}{|l|}{ Paclitaxel } & \multicolumn{5}{|c|}{ Methotrexate } \\
\hline & RMAPC & 0.020 & 0.081 & 0.326 & 1.303 & RMAPC & 0.001 & 0.004 & 0.014 & 0.057 \\
\hline & MCS & 48.119 & 29.999 & 24.653 & 23.148 & MCS & 76.618 & 44.824 & 32.924 & 29.314 \\
\hline & SD & 28.435 & 17.418 & 12.869 & 13.364 & SD & 22.796 & 25.843 & 15.590 & 16.455 \\
\hline \multirow[t]{16}{*}{ Group 2} & \multicolumn{5}{|l|}{ Gemcitabin } & \multicolumn{5}{|c|}{ Dactinomycin } \\
\hline & RMAPC & 0.035 & 0.140 & 0.561 & 2.242 & RMAPC & 0.066 & 0.262 & 1.050 & 4.200 \\
\hline & MCS & 49.354 & 38.411 & 27.808 & 23.784 & MCS & 86.206 & 54.700 & 20.520 & 16.465 \\
\hline & SD & 34.718 & 30.034 & 16.945 & 12.153 & SD & 16.701 & 22.386 & 13.642 & 8.370 \\
\hline & \multicolumn{5}{|l|}{ 5-Flurouracil } & \multicolumn{5}{|c|}{ Vinorelbine } \\
\hline & RMAPC & 0.012 & 0.047 & 0.188 & 0.752 & RMAPC & 0.156 & 0.625 & 2.501 & 10.005 \\
\hline & MCS & 74.861 & 58.001 & 42.337 & 30.505 & MCS & 82.833 & 42.823 & 25.176 & 24.683 \\
\hline & SD & 23.686 & 22.987 & 21.370 & 20.645 & SD & 18.131 & 23.930 & 16.506 & 14.634 \\
\hline & \multicolumn{5}{|l|}{ Doxorubicin } & \multicolumn{5}{|c|}{ Daunorubicin } \\
\hline & RMAPC & 0.028 & 0.112 & 0.447 & 1.789 & RMAPC & 0.133 & 0.531 & 2.126 & 8.502 \\
\hline & MCS & 82.503 & 52.477 & 28.292 & 11.499 & MCS & 83.171 & 61.159 & 25.940 & 20.439 \\
\hline & SD & 21.753 & 24.867 & 22.314 & 10.301 & SD & 21.886 & 25.402 & 23.933 & $17.96 \mid$ \\
\hline & \multicolumn{5}{|l|}{ Etoposide } & \multicolumn{5}{|l|}{ Docetaxel } \\
\hline & RMAPC & 0.130 & 0.521 & 2.083 & 8.333 & RMAPC & 0.260 & 1.042 & 4.166 & 16.665 \\
\hline & MCS & 56.281 & 35.375 & 25.315 & 16.778 & MCS & 32.990 & 23.036 & 23.866 & 26.331 \\
\hline & SD & 21.045 & 19.983 & 15.497 & 11.166 & SD & 22.690 & 17.587 & 16.810 & 19.810 \\
\hline \multirow[t]{8}{*}{ Group 3} & \multicolumn{5}{|l|}{ Cytarabine } & \multicolumn{5}{|c|}{ 6-Mercaptopurine } \\
\hline & RMAPC & 0.121 & 0.482 & 1.929 & 7.716 & RMAPC & 0.543 & 2.170 & 8.680 & 34.720 \\
\hline & MCS & 87.321 & 79.450 & 48.255 & 40.124 & MCS & 84.450 & 78.834 & 62.325 & 55.419 \\
\hline & SD & 17.396 & 22.850 & 26.423 & 26.315 & SD & 22.147 & 16.514 & 29.820 & 32.858 \\
\hline & \multicolumn{5}{|l|}{ Oxaliplatin } & \multicolumn{5}{|l|}{ Cladribine } \\
\hline & RMAPC & 0.020 & 0.081 & 0.325 & 1.301 & RMAPC & 0.366 & 1.462 & 5.850 & 23.399 \\
\hline & MCS & 87.951 & 84.477 & 83.331 & 73.511 & MCS & 102.832 & $101.24 \mid$ & 72.497 & 52.237 \\
\hline & SD & 21.010 & 26.545 & 21.839 & 30.744 & SD & 8.130 & 11.160 & 26.804 & 29.545 \\
\hline
\end{tabular}


Table 2: The average Mean Cell Survival (MCS) of the 17 LCLs at different drug concentrations, expressed as the Ratio of Maximum Achieved Plasma Concentration (RMAPC) (Continued)

\begin{tabular}{|c|c|c|c|c|c|c|c|c|c|}
\hline \multicolumn{5}{|c|}{ Asparaginase } & \multicolumn{5}{|c|}{ Chlorambucil } \\
\hline RMAPC & 0.015 & 0.058 & 0.232 & 0.930 & RMAPC & 2.646 & 10.586 & 42.342 & 169.370 \\
\hline MCS & 92.021 & 91.570 & 90.371 & 82.978 & MCS & 89.903 & 71.879 & 13.225 & 11.099 \\
\hline SD & 13.718 & 13.222 & 18.153 & 15.919 & SD & 17.578 & 26.506 & 10.994 & 7.527 \\
\hline \multicolumn{5}{|c|}{ Cyclophosphamide } & \multicolumn{5}{|c|}{ Topotecan } \\
\hline RMAPC & 0.014 & 0.056 & 0.224 & 0.896 & RMAPC & 6.194 & 24.777 & 99.107 & 396.429 \\
\hline MCS & 86.670 & 93.412 & 89.498 & 84.041 & MCS & 93.897 & 72.802 & 23.250 & 24.784 \\
\hline SD & 21.910 & 18.833 & 17.832 & 17.920 & SD & 19.406 & 21.275 & 16.659 & 21.380 \\
\hline \multicolumn{5}{|c|}{ Ifosphamide } & \multicolumn{5}{|c|}{ Carboplatin } \\
\hline RMAPC & 0.009 & 0.037 & 0.147 & 0.588 & RMAPC & 2.814 & 11.257 & 45.027 & 180.108 \\
\hline MCS & 92.729 & 95.906 & 90.101 & 88.518 & MCS & 91.805 & 79.175 & 77.962 & 52.322 \\
\hline SD & 16.694 & 14.965 & 15.299 & 16.677 & SD & 16.670 & 30.927 & 22.065 & 31.295 \\
\hline \multicolumn{5}{|c|}{ Hydroxyurea } & \multicolumn{5}{|c|}{ Bleomycin } \\
\hline RMAPC & 0.031 & 0.124 & 0.496 & 1.984 & RMAPC & 2.078 & 8.311 & 33.245 & 132.979 \\
\hline MCS & 95.118 & 96.489 & 86.340 & 68.134 & MCS & 90.007 & 88.553 & 82.168 & 69.803 \\
\hline SD & 19.595 & 13.933 & 19.759 & 24.782 & SD & 19.294 & 17.678 & 19.720 & 16.686 \\
\hline \multicolumn{5}{|c|}{ Vinblastine } & \multicolumn{5}{|c|}{ Streptozotocin } \\
\hline RMAPC & 2.603 & 10.413 & 41.650 & 166.600 & RMAPC & \multicolumn{4}{|c|}{ no Cmax data was found } \\
\hline MCS & 81.507 & 53.331 & 28.885 & 21.323 & MCS & 95.430 & 92.335 & 89.919 & 87.805 \\
\hline SD & 25.921 & 25.678 & 17.941 & 15.889 & SD & 15.828 & 18.710 & 14.094 & 22.637 \\
\hline \multicolumn{5}{|c|}{ Bortezomib } & \multicolumn{5}{|l|}{ MG-132 } \\
\hline RMAPC & 0.162 & 0.648 & 2.590 & 10.361 & RMAPC & not used in & al practice & & \\
\hline MCS & 93.119 & 81.671 & 75.498 & 28.700 & MCS & 87.345 & 96.374 & 88.097 & 27.045 \\
\hline SD & 13.779 & 21.572 & 19.617 & 26.948 & SD & 13.980 & 17.493 & 16.974 & 24.310 \\
\hline \multicolumn{10}{|c|}{ Prednisolone } \\
\hline RMAPC & 4.8 & 19.23077 & 76.92308 & 307.6923 & & & & & \\
\hline MCS & 75.55877 & 76.25595 & 62.46537 & 37.23379 & & & & & \\
\hline SD & 4.647172 & | I.0909| & $|3.9702|$ & $11.654 \mid 4$ & & & & & \\
\hline
\end{tabular}

Group I. Highly effective drugs: RMAPC $<0.3$; MCS $<30 \%$

Group 2. Partially effective drugs: RMAPC between 0.3 and I, MCS $<60 \%$

Group 3. Ineffective drugs: RMAPC > I or MCS $>60 \%$

SD: standard deviation in mean cell survival 


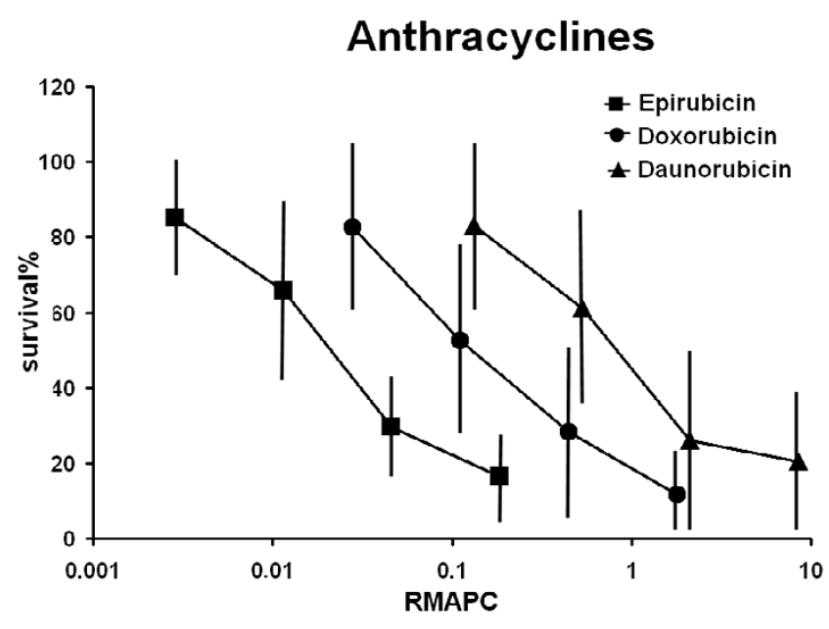

Vinca alkaloids

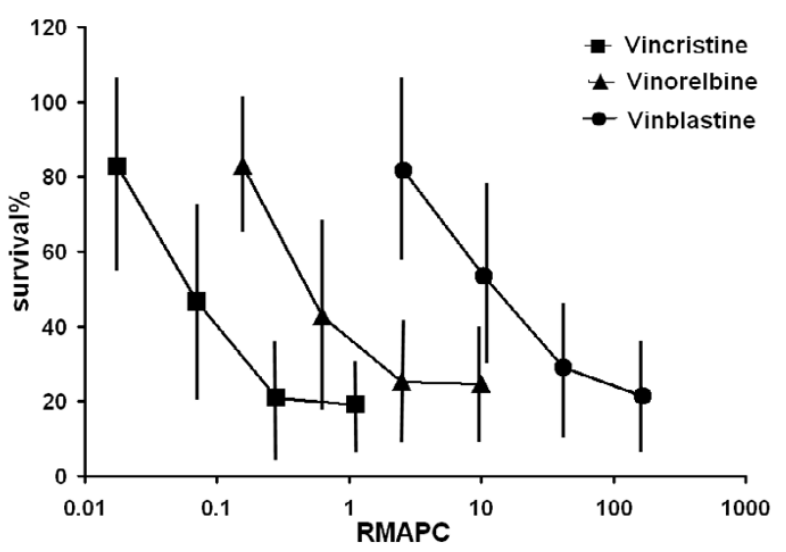

Taxanes

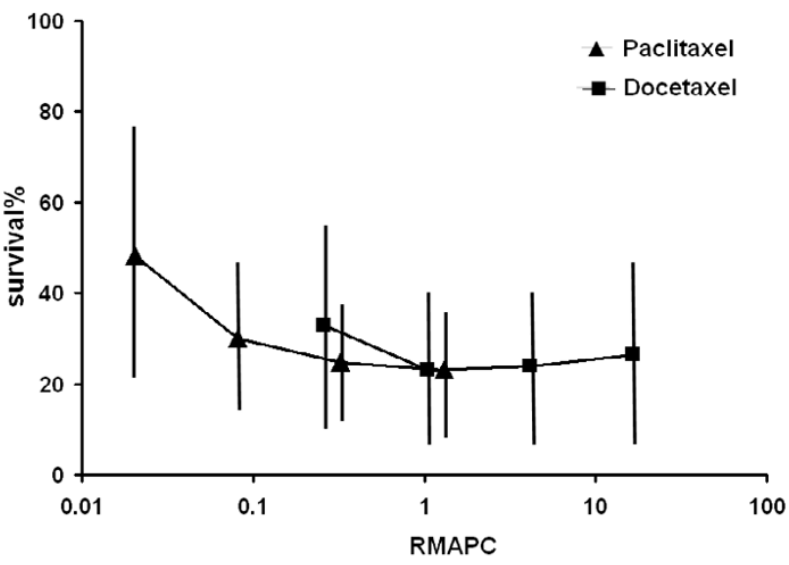

Figure 3

Drug sensitivity of LCLs for anthracyclines, vinca alkaloids and taxans. inhibitory effects on other cell lines or primary tumors (data not shown).

No RMAPC could be determined for streptozocin because no pharmacokinetical trials with established Cmax plasma levels were found. RMAPC was also not available for MG132 because it has not been used clinically. Streptozocin had no effect on survival at the concentrations used in the study. Although at the highest concentrations the proteasome inhibitor MG132 effectively decreased the survival of the cells, its clinically licensed functional homologue, bortezomib, was not effective.

An alternative way to calculate relationship between the in vitro drug concentrations and the in vivo used values is to use the area under curve (AUC) values of the individual drugs. To compare AUC values with the RMAPC data the following formula was used:

in vitro used concentration $(\mu \mathrm{g} / \mathrm{ml}) \times 72 \mathrm{~h} /$ AUC $(\mu \mathrm{gxh} /$ $\mathrm{ml})$ in vivo.

In vivo AUC levels from the literature are summarized in Table 1. Using the formula for representing the mean cell survival curves for all the drugs along a common "ratio of AUC" axis, carboplatin, methotrexate and dactinomycin showed higher efficacy in comparison to RMAPC figures whereas the sensitivity order for the other drugs or for the group classifications did not change.

\section{Discussion}

The presented data suggest that many EBV transformed Bcell lines share a common cytotoxic drug sensitivity profile independent of their origin. This profile does not change even after many years of in vitro culturing. EBV appears to be the necessary and sufficient etiological agent behind the malignant immunoblastic B-cell lymphoproliferations in immunosuppressed patients. In all these cases the EBV encoded, latency associated viral proteins drive the cell proliferation without any obvious need for additional genetic changes. The phenotype of these tumors closely resembles the in vitro growing LCLs and the experimentally induced tumors that appeared upon intraperitoneal implantation of LCLs into SCID mice [11]. Considering the phenotypic and karyotypic stability of EBV transformed B-cells, our data raises the hope that PTLDs, AIDS associated CNS lymphomas and XLP associated lymphoproliferations may show similar patterns of drug sensitivity as the ones that we have established in the present study on a cohort of diverse LCLs.

Sugimoto et al. suggested that EBV mediated transformation is a two step process where, after prolonged passage, the cells may become aneuploid, accumulate p53 mutations, down regulate the p16/retinoblastoma protein 

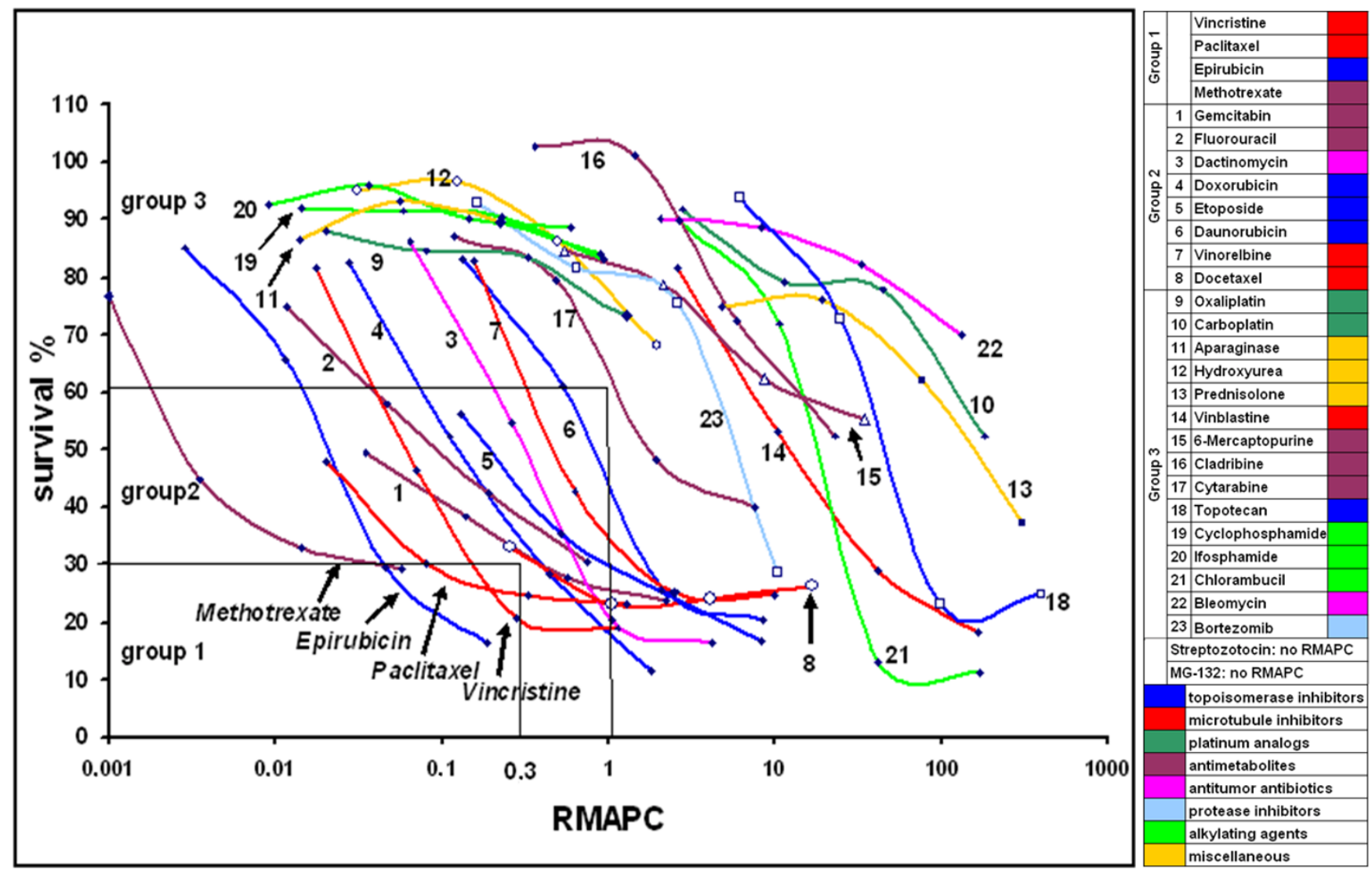

Figure 4

Drug sensitivity mean values of 17 lymphoblastoid cell lines plotted against the Ratio of Maximum Achieved Plasma Concentration (RMAPC) values. The different drug families are labelled with different colours. The highly effective (Group I) and partially effective (Group 2) drugs are identified.

pathway and become tumorigenic upon subcutaneous inoculation into nude mice [7]. Some of the cell lines that we have examined were kept in continuous culture over five years (IARC171, LSspontan, 980215). These cells are still euploid and show low soft agar clonability. The oldest line IARC171, has wild type p53, is non-tumorigenic upon subcutan inoculation but produces generalized immunoblastic lymphomas in SCID mice after intraperitoneal inoculation. The findings that PTLD can arise very rapidly in post-transplant patient together with the data that freshly EBV infected B-cells can grow into generalized immunoblastic lymphomas within a few weeks, implies that the development of PTLD does not require aneuploidy. Sawada et al. reported that LCLs with negative or low telomerase activity and normal karyotypes are more sensitive against certain drugs, than LCLs with a high telomerase activity and abnormal karyotypes [22]. This data together with our present findings may suggest that rap- idly emerging PTLDs may show different drug sensitivity pattern from EBV positive aneuploid diffuse large cell Bcell lymphomas that arise for example in AIDS patients in sub-Saharan Africa.

Our data shows that euploid LCLs are particularly sensitive for anti-microtubule drugs and anthracyclines. Although all LCLs showed a dose dependent increase of cytotoxicity when treated with different members of these two drug families, only vincristine and paclitaxel as well as epirubicin and doxorubicin were considered to be effective when the used in vitro drug concentrations were compared to the maximum achievable plasma concentrations.

Alkylating agents, such as cyclophosphamide and ifosphamide were not effective on LCLs. This might be explained by the fact that both of these compounds are 
pro-drugs that have to be converted into active metabolites by the liver in vivo. Prednisolone was active but only in very high concentrations.

\section{Conclusion}

Vincristine and methotrexate are included in the frequently used CHOP and VAPEC-regimes, but no data could be found for the clinical use of epirubicin and paclitaxel for the treatment of PTLD or other EBV induced lymphoproliferations. Our results suggest that the inclusion of epirubicin and paclitaxel into chemotherapy protocols against PTLD may be justified.

\section{Competing interests}

The author(s) declare that they have no competing interests.

\section{Authors' contributions}

The project was conceived and designed by LS. The experiments were mainly carried out and/or coordinated by LM. GS took part in cell culturing, and preparation of the microtiter plates for in vitro drug sensitivity assays. EF was responsible for measuring the plates using the automated laser confocal fluorescent microscope. LS and EF wrote the computer programs QuantCapture 4.0 and QuantCount 3.0. LM and HS analysed and interpreted the data. SE made comparable the in vitro results with the in vivo data. AGJ, EO, HS together with the other authors have been involved in the planning of the experimental details, and the drafting and critical reading of the manuscript. All authors read and approved the final manuscript.

\section{Acknowledgements}

We thank the Swedish Cancer Society, the Swedish Research Council, Child Cancer Foundation and the IRIS center for economic support. We thank Doctor Noemi Nagy for the TR cell line.

\section{References}

I. Bower M: The management of lymphoma in the immunosuppressed patient. Best Pract Res Clin Haematol 2002, I 5(3):5 I7-532.

2. Leblond V, Sutton L, Dorent R, Davi F, Bitker MO, Gabarre J, Charlotte F, Ghoussoub J], Fourcade C, Fischer A, et al.: Lymphoproliferative disorders after organ transplantation: a report of 24 cases observed in a single center. J Clin Oncol 1995, I3(4):96I-968.

3. Opelz G, Dohler B: Lymphomas after solid organ transplantation: a collaborative transplant study report. Am J Transplant 2004, 4(2):222-230.

4. Orazi A, Hromas RA, Neiman RS, Greiner TC, Lee CH, Rubin L, Haskins S, Heerema NA, Gharpure V, Abonour R, Srour EF, Cornetta $\mathrm{K}$ : Post transplantation lymphoproliferative disorders in bone marrow transplant recipients are aggressive diseases with a high incidence of adverse histologic and immunobiologic features. Am J Clin Pathol 1997, I07(4):419-429.

5. Gross TG, Loechelt BJ: Epstein-Barr virus associated disease following blood or marrow transplant. Pediatr Transplant 2003, 7(Suppl 3):44-50.

6. Andersson JP: Clinical aspects on Epstein-Barr virus infection. Scand J Infect Dis Suppl 1991, 80:94-104.

7. Sugimoto $M$, Tahara $H$, Ide T, Furuichi $Y$ : Steps involved in immortalization and tumorigenesis in human B-lymphoblastoid cell lines transformed by Epstein-Barr virus. Cancer Res 2004, 64(10):336I-3364.
8. Rickinson AB, Murray RJ, Brooks J, Griffin H, Moss DJ, Masucci MG: $T$ cell recognition of Epstein-Barr virus associated lymphomas. Cancer Surv 1992, 13:53-80.

9. Masucci MG, Gavioli R, de Campos-Lima PO, Zhang QJ, Trivedi P, Dolcetti R: Transformation-associated Epstein-Barr virus antigens as targets for immune attack. Ann N Y Acad Sci 1993, 690:86-100.

10. Masucci MG, Ernberg I: Epstein-Barr virus: adaptation to a life within the immune system. Trends Microbiol 1994, 2(4): I25-I30.

II. Lacerda JF, Ladanyi M, Louie DC, Fernandez JM, Papadopoulos EB, O'Reilly RJ: Human Epstein-Barr virus (EBV)-specific cytotoxic $T$ lymphocytes home preferentially to and induce selective regressions of autologous EBV-induced B cell lymphoproliferations in xenografted C.B-I 7 scid/scid mice. J Exp Med 1996, 183(3): 1215-1228.

12. Rowe M, Young LS, Crocker J, Stokes $\mathrm{H}$, Henderson S, Rickinson AB: Epstein-Barr virus (EBV)-associated lymphoproliferative disease in the SCID mouse model: implications for the pathogenesis of EBV-positive lymphomas in man. J Exp Med 199I, 173(1):147-158.

13. Gross TG, Steinbuch M, DeFor T, Shapiro RS, McGlave P, Ramsay NK, Wagner JE, Filipovich AH: B cell lymphoproliferative disorders following hematopoietic stem cell transplantation: risk factors, treatment and outcome. Bone Marrow Transplant 1999, 23(3):25I-258.

14. Curtis RE, Travis LB, Rowlings PA, Socie G, Kingma DW, Banks PM, Jaffe ES, Sale GE, Horowitz MM, Witherspoon RP, Shriner DA, Weisdorf DJ, Kolb HJ, Sullivan KM, Sobocinski KA, Gale RP, Hoover RN, Fraumeni JF Jr., Deeg HJ: Risk of lymphoproliferative disorders after bone marrow transplantation: a multi-institutional study. Blood 1999, 94(7):2208-2216.

15. Zutter MM, Martin PJ, Sale GE, Shulman HM, Fisher L, Thomas ED, Durnam DM: Epstein-Barr virus lymphoproliferation after bone marrow transplantation. Blood 1988, 72(2):520-529.

16. Gustafsson A, Levitsky V, Zou JZ, Frisan T, Dalianis T, Ljungman P, Ringden O, Winiarski J, Ernberg I, Masucci MG: Epstein-Barr virus (EBV) load in bone marrow transplant recipients at risk to develop posttransplant lymphoproliferative disease: prophylactic infusion of EBV-specific cytotoxic T cells. Blood 2000, 95(3):807-8।4.

17. Armitage JM, Kormos RL, Stuart RS, Fricker FJ, Griffith BP, Nalesnik M, Hardesty RL, Dummer JS: Posttransplant lymphoproliferative disease in thoracic organ transplant patients: ten years of cyclosporine-based immunosuppression. J Heart Lung Transplant 1991, I 0(6):877-86; discussion 886-7.

18. Milpied N, Vasseur B, Parquet N, Garnier JL, Antoine C, Quartier P, Carret AS, Bouscary D, Faye A, Bourbigot B, Reguerre Y, Stoppa AM, Bourquard P, Hurault de Ligny B, Dubief F, Mathieu-Boue A, Leblond $\checkmark$ : Humanized anti-CD20 monoclonal antibody (Rituximab) in post transplant B-lymphoproliferative disorder: a retrospective analysis on 32 patients. Ann Oncol 2000, II Suppl I:II3-116.

19. Savoldo B, Rooney CM, Quiros-Tejeira RE, Caldwell Y, Wagner HJ, Lee T, Finegold MJ, Dotti G, Heslop HE, Goss JA: Cellular immunity to Epstein-Barr virus in liver transplant recipients treated with rituximab for post-transplant lymphoproliferative disease. Am J Transplant 2005, 5(3):566-572.

20. Taylor AL, Marcus R, Bradley JA: Post-transplant lymphoproliferative disorders (PTLD) after solid organ transplantation. Crit Rev Oncol Hematol 2005, 56(I): I55-167.

21. Muti G, Cantoni S, Oreste P, Klersy C, Gini G, Rossi V, D'Avanzo G, Comoli P, Baldanti F, Montillo M, Nosari A, Morra E: Post-transplant lymphoproliferative disorders: improved outcome after clinico-pathologically tailored treatment. Haematologica 2002, 87(1):67-77.

22. Sawada K, Noda K, Nakajima H, Shimbara N, Furuichi Y, Sugimoto $M$ : Differential cytotoxicity of anticancer agents in pre- and post-immortal lymphoblastoid cell lines. Biol Pharm Bull 2005, 28(7): | 202-1207.

23. Fogli S, Danesi R, Gennari A, Donati S, Conte PF, Del Tacca M: Gemcitabine, epirubicin and paclitaxel: pharmacokinetic and pharmacodynamic interactions in advanced breast cancer. Ann Oncol 2002, I3(6):919-927.

24. Toffoli G, Corona G, Cattarossi G, Boiocchi M, Di Gennaro G, Tirelli $U$, Vaccher E: Effect of highly active antiretroviral therapy (HAART) on pharmacokinetics and pharmacodynamics of 
doxorubicin in patients with HIV-associated non-Hodgkin's lymphoma. Ann Oncol 2004, I 5(I 2): 1805-1809.

25. Minami H, Ando Y, Sakai S, Shimokata K: Clinical and pharmacologic analysis of hyperfractionated daily oral etoposide. J Clin Oncol 1995, I3(1):191-199.

26. Chan GL, Erdmann GR, Gruber SA, Stock P, Chen S, Ascher NL, Canafax DM: Pharmacokinetics of 6-thiouric acid and 6-mercaptopurine in renal allograft recipients after oral administration of azathioprine. Eur J Clin Pharmacol 1989, 36(3):265-27I.

27. Gerrits CJ, Schellens JH, Burris H, Eckardt JR, Planting AS, van der Burg ME, Rodriguez Gl, Loos WJ, van Beurden V, Hudson I, Von Hoff DD, Verweij ]: A comparison of clinical pharmacodynamics of different administration schedules of oral topotecan (Hycamtin). Clin Cancer Res 1999, 5( I):69-75.

28. Rischin D, Ackland SP, Smith J, Garg MB, Clarke S, Millward MJ, Toner GC, Zalcberg J: Phase I and pharmacokinetic study of docetaxel in combination with epirubicin and cyclophosphamide in advanced cancer: dose escalation possible with granulocyte colony-stimulating factor, but not with prophylactic antibiotics. Ann Oncol 2002, I3(II): 1810-1818.

29. Terashi K, Oka M, Soda H, Fukuda M, Kawabata S, Nakatomi K, Shiozawa K, Nakamura T, Tsukamoto K, Noguchi Y, Suenaga M, Tei C Kohno S: Interactions of ofloxacin and erythromycin with the multidrug resistance protein (MRP) in MRP-overexpressing human leukemia cells. Antimicrob Agents Chemother 2000, 44(6): $1697-1700$.

30. Desai ZR, Van den Berg HW, Bridges JM, Shanks RG: Can severe vincristine neurotoxicity be prevented? Cancer Chemother Pharmacol |982, 8(2):2||-2|4.

31. Stewart DJ, Lu K, Benjamin RS, Leavens ME, Luna M, Yap HY, Loo TL: Concentration of vinblastine in human intracerebral tumor and other tissues. J Neurooncol 1983, I(2): I39-144.

32. Bates SE, Bakke S, Kang M, Robey RW, Zhai S, Thambi P, Chen CC, Patil S, Smith T, Steinberg SM, Merino M, Goldspiel B, Meadows B, Stein WD, Choyke P, Balis F, Figg WD, Fojo T: A phase I/II study of infusional vinblastine with the P-glycoprotein antagonist valspodar (PSC 833) in renal cell carcinoma. Clin Cancer Res 2004, I (14):4724-4733.

33. Zhou XJ, Bore P, Monjanel S, Sahnoun Z, Favre R, Durand A, Rahmani $R$ : Pharmacokinetics of navelbine after oral administration in cancer patients. Cancer Chemother Pharmacol I99I, 29(I):66-70.

34. Freyer G, Delozier T, Lichinister M, Gedouin D, Bougnoux P, His $P$, Imadalou K, Trillet-Lenoir V: Phase II study of oral vinorelbine in first-line advanced breast cancer chemotherapy. J Clin Oncol 2003, 2 I (I):35-40.

35. Ghazal-Aswad S, Calvert AH, Newell DR: A single-sample assay for the estimation of the area under the free carboplatin plasma concentration versus time curve. Cancer Chemothe Pharmacol 1996, 37(5):429-434.

36. Gamelin E, Bouil AL, Boisdron-Celle M, Turcant A, Delva R, Cailleux A, Krikorian A, Brienza S, Cvitkovic E, Robert J, Larra F, Allain P: Cumulative pharmacokinetic study of oxaliplatin, administered every three weeks, combined with 5-fluorouracil in colorectal cancer patients. Clin Cancer Res 1997, 3(6):89 I-899.

37. Graham MA, Lockwood GF, Greenslade D, Brienza S, Bayssas M, Gamelin E: Clinical pharmacokinetics of oxaliplatin: a critical review. Clin Cancer Res 2000, 6(4): 1205-1218.

38. Bacci G, Loro L, Longhi A, Bertoni F, Bacchini P, Versari M, Picci P Serra M: No correlation between methotrexate serum leve and histologic response in the pre-operative treatment of extremity osteosarcoma. Anticancer Drugs 2006, I7(4):4II-4I5.

39. Crews KR, Liu T, Rodriguez-Galindo C, Tan M, Meyer WH, Panetta JC, Link MP, Daw NC: High-dose methotrexate pharmacokinetics and outcome of children and young adults with osteosarcoma. Cancer 2004, 100(8): 1724-1733.

40. Albertioni F, Lindemalm S, Reichelova V, Pettersson B, Eriksson S, Juliusson G, Liliemark J: Pharmacokinetics of cladribine in plasma and its 5'-monophosphate and 5'-triphosphate in leukemic cells of patients with chronic lymphocytic leukemia. Clin Cancer Res 1998, 4(3):653-658.

4I. Casale F, Canaparo R, Serpe L, Muntoni E, Pepa CD, Costa M, Mairone L, Zara GP, Fornari G, Eandi M: Plasma concentrations of 5 fluorouracil and its metabolites in colon cancer patients. Pharmacol Res 2004, 50(2): $173-179$.

42. DeAngelis LM, Kreis W, Chan K, Dantis E, Akerman S: Pharmacokinetics of ara-C and ara- $U$ in plasma and CSF after high-dose administration of cytosine arabinoside. Cancer Chemother Pharmacol 1992, 29(3): 173-177.

43. Broughton A, Strong JE, Holoye PY, Bedrossian CW: Clinical pharmacology of bleomycin following intravenous infusion as determined by radioimmunoassay. Cancer 1977, 40(6):2772-2778.

44. Peng YM, Alberts DS, Chen HS, Mason N, Moon TE: Antitumour activity and plasma kinetics of bleomycin by continuous and intermittent administration. $\mathrm{Br}$ / Cancer 1980, 4 I (4):644-647.

45. Veal GJ, Cole M, Errington J, Parry A, Hale J, Pearson AD, Howe K, Chisholm JC, Beane C, Brennan B, Waters F, Glaser A, Hemsworth $S$, McDowell H, Wright Y, Pritchard-Jones K, Pinkerton R, Jenner G, Nicholson J, Elsworth AM, Boddy AV: Pharmacokinetics of dactinomycin in a pediatric patient population: a United Kingdom Children's Cancer Study Group Study. Clin Cancer Res 2005, I I ( 16):5893-5899.

46. Papandreou CN, Daliani DD, Nix D, Yang H, Madden T, Wang $X$, Pien CS, Millikan RE, Tu SM, Pagliaro L, Kim J, Adams J, Elliott P, Esseltine D, Petrusich A, Dieringer P, Perez C, Logothetis CJ: Phase I trial of the proteasome inhibitor bortezomib in patients with advanced solid tumors with observations in androgen-independent prostate cancer. J Clin Oncol 2004, 22(II):2108-2I2I.

47. Xie H, Griskevicius L, Stahle L, Hassan Z Yasar U, Rane A, Broberg $U$, Kimby E, Hassan M: Pharmacogenetics of cyclophosphamide in patients with hematological malignancies. Eur J Pharm Sci 2006, 27(I):54-6I.

48. Wright JE, Elias A, Tretyakov O, Holden S, Andersen J, Wheeler C, Schwartz G, Antman K, Rosowsky A, Frel E 3rd, et al.: High-dose ifosfamide, carboplatin, and etoposide pharmacokinetics: correlation of plasma drug levels with renal toxicity. Cancer Chemother Pharmacol 1995, 36(4):345-35 I.

49. GlaxoSmithKline Research Triangle Park NC: Prescribing Information Leukeran $®$. http://us.gsk.com/products/assets/ us leukeran.pdf.

50. Ho DH, Yap HY, Brown N, Benjamin RS, Friereich EJ, Blumenschein GR, Bodey GP: Clinical pharmacology of intramuscularly administered L-asparaginase. J Clin Pharmacol I98I, 2I (2):72-78.

51. Ylikangas P, Mononen I: Serious neutropenia in ALL patients treated with L-asparaginase may be avoided by therapeutic monitoring of the enzyme activity in the circulation. Ther Drug Monit 2002, 24(4):502-506.

52. Gwilt PR, Manouilov KK, McNabb J, Swindells SS: Pharmacokinetics of hydroxyurea in plasma and cerebrospinal fluid of HIV. I-infected patients. J Clin Pharmacol 2003, 43(9): 1003-1007.

53. Rostin M, Barthe P, Houin G, Alvinerie M, Bouissou F: Pharmacokinetics of prednisolone in children with the nephrotic syndrome. Pediatr Nephrol 1990, 4(5):470-473.

\section{Pre-publication history}

The pre-publication history for this paper can be accessed here:

http://www.biomedcentral.com/1471-2407/6/265/pre pub

Publish with Bio Med Central and every scientist can read your work free of charge

"BioMed Central will be the most significant development for disseminating the results of biomedical research in our lifetime. "

Sir Paul Nurse, Cancer Research UK

Your research papers will be:

- available free of charge to the entire biomedical community

- peer reviewed and published immediately upon acceptance

- cited in PubMed and archived on PubMed Central

- yours - you keep the copyright
BioMedcentral 